Agrotrópica 28(2): 179 - 184. 2016.

Centro de Pesquisas do Cacau, Ilhéus, Bahia, Brasil

\title{
VARIABILIDADE POPULACIONAL NA PRODUÇÃO DE ÓLEO, LIPÍDIOS TOTAIS NA AMÊNDOA E POLPA DE TUCUMÃ COLETADOS NO ESTADO DO ACRE
}

\author{
Rean Augusto Zaninetti', Adônis Moreira², Antônio Sergio Ferraudo \\ Sandra Tereza Teixeira ${ }^{4}$ \\ ${ }^{1}$ Universidade Federal do Acre, 69920-900, Rio Branco, Acre, Brasil; ${ }^{2}$ Embrapa Soja, 86001-970, Londrina, Paraná, Brasil; \\ ${ }^{3}$ Universidade Estadual Paulista Júlio de Mesquita Filho, Faculdade de Ciências Agrárias e Veterinárias de Jaboticabal, \\ 14884-900, Jaboticabal, São Paulo, Brasil; ${ }^{4}$ Faculdade Meta, 69919-230, Rio Branco, Acre, Brasil. \\ adonismoreira66@gmail.com
}

\begin{abstract}
Na Amazônia são encontradas espécies cujos frutos apresentam quantidade de óleo com valor econômico para diferentes aplicações industriais. Este trabalho teve o objetivo de avaliar o potencial de produção de óleo do tucumã (Astrocaryum aculeatum) para a produção de biodiesel em diferentes áreas do Estado do Acre. As variáveis avaliadas para a caracterização foram: produção, quantidade de polpa (epicarpo+mesocarpo), castanha (endocarpo), amêndoas (semente do interior do endocarpo), teores de lipídios totais na amêndoa e na polpa e os atributos químicos do solo. O teor de lipídios na amêndoa foi de $21,9 \%$, enquanto na polpa foi de $31,9 \%$. Apesar da diversidade genética, o tucumã é uma espécie que possui boa produtividade em óleo, mesmo em solos ácidos e com baixa fertilidade, apresentando potencial para ser utilizado como uma fonte adicional de renda pelas comunidades amazonidas.
\end{abstract}

Palavras-chave: biocombustivel, Amazônia, fertilidade do solo, diversidade genética

\footnotetext{
Population variability in production of oil, total lipids in kernel and pulp of tucumã (Astrocaryum aculeatum) collected in the Acre State, Brazil. In the Amazon region species are found whose fruits have quantities of oil with economic value for various industrial applications. This study had the objective to evaluate the potential of oils extracted from tucumã palm fruit (Astrocaryum aculeatum) found in different areas of the Acre State. The yield, amount of pulp (mesocarp + epicarp), seed (endocarp), kernel (seed inside the endocarp), total lipid (almond and the pulp), and chemical soil properties were evaluated. The content of lipids in kernel was $21.9 \%$, while the pulp was $31.9 \%$. Despite the genetic diversity, the tucumã palm fruit is a species that has good bio-oil yield, even in acid soils with low fertility, with potential to be used as an additional source of income for communities in the Amazon region.
}

Key words: biofuel, Amazon soil fertility, genetic diversity 


\section{Introdução}

A alta biodiversidade da Amazônia tem sido objeto de pesquisa, com resultados diversos sobre seu potencial de uso das plantas nativas da região (Schroth, 2004; Clement et al., 2005). Verificou-se que os mais promissores são os frutos e sementes para produção de biocombustível oriundos da mamoninha do mato (Mabea fistulifera), o babaçú (Orbignya phalerata, Mart.) e a palmeira de tucumã ou tucumanzeiro (Clement \& Arkcoll 1991; Clement et al. 2005; Pereira 2007).

No caso do tucumanzeiro, palmeira monocaule com ocorrência dispersa nos estados do Acre, Amazonas, Rondônia, Pará, Mato Grosso e Roraima (Lorenzi et al. 1996; Shanley e Medina 2005), pode ser encontrada em pequenas densidades no interior da floresta em populações espontâneas, com ocorrência mais abundante em áreas desmatadas, formando grandes e densas manchas em roçados, pastagens degradadas, capoeiras (floresta secundária) e margens de estradas (Cavalcante 1996; Miranda et al. 2001; Barcelar-Lima et al. 2006). Devido à ausência de material selecionado, abundância de plantas nas condições descritas e a baixa germinação das sementes, os plantios comerciais na região são escassos (Schroth et al., 2004)

Os frutos são produzidos durante o ano todo, porém o pico da produção ocorre nos meses de dezembro a abril (Schroth et al., 2004). Na região Norte, sua importância econômica baseia-se principalmente na exploração da polpa dos frutos, que pode ser consumida in natura ou na forma de sorvete, suco, licor e doce (Schroth et al., 2004). Da polpa dos frutos e das sementes podem ser extraídos diferentes tipos de óleos comestíveis e ser utilizada na suplementação alimentar humana e na fabricação de ração animal (Mendonça, 1996).

Este trabalho teve como objetivo avaliar e identificar a influência dos atributos químicos do solo sobre a quantidade de lipídios totais presente na amêndoa e na polpa de frutos de tucumã localizados no entorno do município de Rio Branco, Estado do Acre.

\section{Material e Métodos}

A colheita dos frutos de tucumã foi realizada nas margens das BR-364 e BR-317, a uma distância de até $150 \mathrm{~km}$ da cidade de Rio Branco, Estado do Acre
( $9^{\circ} 59^{\prime} \mathrm{LS}$ e $\left.67^{\circ} 48^{\prime} \mathrm{LO}\right)$. O clima na região é tropical úmido, com temperatura média de $25^{\circ} \mathrm{C}$ e precipitação anual de $1950 \mathrm{~mm}$ e altitude entre 140 a 220 metros. Os locais de coleta foram selecionados pela presença de um número mínimo de dez plantas e a presença de frutos em pelo menos três delas. O georreferenciamento dos locais foi realizado na parte central de cada população e as coordenadas dos locais são mostradas na Tabela 1. Os pontos de um (1) a nove (9) correspondem aos locais amostrados na BR364 e os pontos de 10 a 22 correspondem aos coletados na BR-317, ambas dentro do Estado do Acre. A colheita foi realizada no período de final do mês de outubro ao começo do mês de dezembro de 2010.

No mês de julho de 2010 foi feita à seleção das áreas, ocasião que frutos estavam ainda verdes. A amostragem de solo foi realizada em cada área de coleta, retirando-se 20 amostras simples na profundidade 0 a $20 \mathrm{~cm}$ com trado holandês para formar uma amostra composta conforme procedimento descrito por Raij et al. (1997). Após secas ao ar,

Tabela 1. Coordenadas geográficas dos pontos de coleta de frutos de plantas de tucumanzeiro

\begin{tabular}{|c|c|c|}
\hline $\begin{array}{c}\text { Áreas de } \\
\text { Amostragem }\end{array}$ & Latitude & Longitude \\
\hline 1 & $10^{\circ} 00^{\circ} 46,0^{\prime \prime} \mathrm{S}$ & $67^{\circ} 42^{\prime} 49,3^{\prime \prime} \mathrm{W}$ \\
\hline 2 & $10^{\circ} 01^{\prime} 31,1^{\prime \prime} \mathrm{S}$ & $67^{\circ} 38^{\prime} 40,5^{\prime \prime} \mathrm{W}$ \\
\hline 3 & $10^{\circ} 03^{\prime} 31,7^{\prime \prime} \mathrm{S}$ & $67^{\circ} 19^{\prime} 24,5^{\prime \prime} \mathrm{W}$ \\
\hline 4 & $10^{\circ} 02^{\prime} 26,6^{\prime \prime} \mathrm{S}$ & $67^{\circ} 15^{\prime} 53,9^{\prime \prime} \mathrm{W}$ \\
\hline 5 & $09^{\circ} 59^{\prime} 40,6^{\prime \prime} \mathrm{S}$ & $67^{\circ} 11^{\prime} 09,2^{\prime \prime} \mathrm{W}$ \\
\hline 6 & $09^{\circ} 58^{\prime} 05,9^{\prime \prime} \mathrm{S}$ & $67^{\circ} 07^{\prime} 26,0^{\prime \prime} \mathrm{W}$ \\
\hline 7 & $09^{\circ} 56^{\prime} 46,6^{\prime \prime} \mathrm{S}$ & $67^{\circ} 03^{\prime} 28,0^{\prime \prime} \mathrm{W}$ \\
\hline 8 & $09^{\circ} 52^{\prime} 01,1^{\prime \prime} \mathrm{S}$ & $66^{\circ} 54^{\prime} 51,7^{\prime \prime} \mathrm{W}$ \\
\hline 9 & $09^{\circ} 54^{\prime} 14,6^{\prime \prime} \mathrm{S}$ & $66^{\circ} 56^{\prime} 48,6^{\prime \prime} \mathrm{W}$ \\
\hline 10 & $10^{\circ} 16^{\prime} 51,0^{\prime \prime} \mathrm{S}$ & $67^{\circ} 41^{\prime} 54,6^{\prime \prime} \mathrm{W}$ \\
\hline 11 & $10^{\circ} 18^{\prime} 33,9^{\prime \prime} \mathrm{S}$ & $67^{\circ} 41^{\prime} 31,3$ "W \\
\hline 12 & $10^{\circ} 21^{\prime} 47,9^{\prime \prime} \mathrm{S}$ & $67^{\circ} 41^{\prime} 37,5^{\prime \prime} \mathrm{W}$ \\
\hline 13 & $10^{\circ} 28^{\prime} 21,6^{\prime \prime} \mathrm{S}$ & $67^{\circ} 40^{\prime} 58,4^{\prime \prime} \mathrm{W}$ \\
\hline 14 & $10^{\circ} 35^{\prime} 08,4^{\prime \prime} \mathrm{S}$ & $67^{\circ} 41^{\prime} 57,6^{\prime \prime} \mathrm{W}$ \\
\hline 15 & $10^{\circ} 35^{\prime} 42,9^{\prime \prime} \mathrm{S}$ & $67^{\circ} 42^{\prime} 41,4^{\prime \prime} \mathrm{W}$ \\
\hline 16 & $10^{\circ} 36^{\prime} 05,2^{\prime \prime} \mathrm{S}$ & $67^{\circ} 43^{\prime} 25,0^{\prime \prime} \mathrm{W}$ \\
\hline 17 & $10^{\circ} 36^{\prime} 11,0^{\prime \prime} \mathrm{S}$ & $67^{\circ} 45^{\prime} 44,6^{\prime \prime} \mathrm{W}$ \\
\hline 18 & $10^{\circ} 36^{\prime} 10,7^{\prime \prime} \mathrm{S}$ & $67^{\circ} 47^{\prime} 12,9^{\prime \prime} \mathrm{W}$ \\
\hline 19 & $10^{\circ} 36^{\prime} 23,9^{\prime \prime} \mathrm{S}$ & $67^{\circ} 47^{\prime} 53,5^{\prime \prime} \mathrm{W}$ \\
\hline 20 & $10^{\circ} 36^{\prime} 29,8^{\prime \prime} \mathrm{S}$ & $67^{\circ} 48^{\prime} 41,4^{\prime \prime} \mathrm{W}$ \\
\hline 21 & $10^{\circ} 35^{\prime} 06,2^{\prime \prime} \mathrm{S}$ & $67^{\circ} 52^{\prime} 07,6^{\prime \prime} \mathrm{W}$ \\
\hline 22 & $10^{\circ} 35^{\prime} 44,5^{\prime \prime} \mathrm{S}$ & $67^{\circ} 57^{\prime} 14,3^{\prime \prime} \mathrm{W}$ \\
\hline
\end{tabular}


destorroadas e passadas em peneiras de $2,0 \mathrm{~mm}$, foi realizada a análise dos atributos químicos do solo (Raij et al., 1997), que consistiu na determinação do $\mathrm{pH}$ $\left(\mathrm{H}_{2} \mathrm{O}\right)$, acidez potencial $\left(\mathrm{H}^{+}+\mathrm{Al}^{3+}\right), \mathrm{Al}^{3+}$ trocável, $\mathrm{P}$ disponível (resina), capacidade de troca de cátions ficando (CTC, " $\mathrm{K}^{+}, \mathrm{Ca}^{2+}, \mathrm{Mg}^{2+}, \mathrm{H}^{+}+\mathrm{Al}^{3+}$ ") e saturação por bases (V\%) (Tabela 2).

Em cada local foram colhidos os frutos retirados dos cachos para contagem e determinação da massa. Foram selecionados, ao acaso, trinta frutos em estágio de amadurecimento e trinta frutos totalmente maduros de cada palmeira. Nas amostras com frutos maduros foram determinados a massa, e posteriormente despolpados e separados em polpa (epicarpo + mesocarpo), castanha (endocarpo) e amêndoas (semente retirada do interior do endocarpo), enquanto os não maduros foram acondicionados em cestos plásticos, permanecendo em temperatura ambiente $\mathrm{e}$ à sombra até atingirem o amadurecimento completo.

A determinação do teor de lipídio total nas amostras de amêndoas, polpa foi realizada com a extração por

Tabela 2. Atributos químicos do solo coletados dos diferentes locais $\operatorname{amostrados}^{1}$

\begin{tabular}{|c|c|c|c|c|c|c|}
\hline \multirow[t]{2}{*}{ Locais } & \multirow{2}{*}{$\begin{array}{c}\mathrm{pH} \\
\mathrm{CaCl}_{2}\end{array}$} & \multirow{2}{*}{$\begin{array}{c}\mathrm{P} \\
\mathrm{mg} \mathrm{dm}^{-3}\end{array}$} & $\mathrm{H}^{+}+\mathrm{Al}^{3}$ & $\mathrm{Al}^{3+}$ & CTC & \multirow{2}{*}{$\begin{array}{l}\mathrm{V} \\
\%\end{array}$} \\
\hline & & & \multicolumn{3}{|c|}{$-\operatorname{mmol}_{\mathrm{c}} \mathrm{dm}^{-3}-$} & \\
\hline 1 & 3,9 & 9 & 88 & 17,0 & 106,6 & 17 \\
\hline 2 & 5,5 & 12 & 28 & 0,0 & 103,7 & 73 \\
\hline 3 & 3,8 & 9 & 64 & 11,0 & 75,4 & 15 \\
\hline 4 & 3,8 & 7 & 150 & 52,0 & 160,9 & 7 \\
\hline 5 & 4,0 & 7 & 52 & 6,0 & 62,7 & 17 \\
\hline 6 & 4,5 & 6 & 47 & 4,0 & 73,4 & 36 \\
\hline 7 & 4,5 & 7 & 47 & 3,0 & 75,2 & 38 \\
\hline 8 & 3,9 & 9 & 109 & 20,0 & 128,0 & 15 \\
\hline 9 & 4,4 & 9 & 72 & 11,0 & 108,4 & 34 \\
\hline 10 & 4,0 & 7 & 64 & 8,0 & 84,3 & 24 \\
\hline 11 & 4,1 & 7 & 52 & 5,0 & 68,0 & 24 \\
\hline 12 & 3,9 & 8 & 72 & 8,0 & 90,5 & 20 \\
\hline 13 & 4,2 & 15 & 58 & 5,0 & 81,7 & 29 \\
\hline 14 & 4,5 & 8 & 52 & 3,0 & 80,1 & 35 \\
\hline 15 & 4,2 & 6 & 47 & 2,0 & 67,3 & 30 \\
\hline 16 & 4,0 & 5 & 38 & 6,0 & 49,0 & 22 \\
\hline 17 & 4,0 & 5 & 42 & 5,0 & 57,1 & 26 \\
\hline 18 & 4,0 & 6 & 47 & 11,0 & 55,1 & 15 \\
\hline 19 & 4,0 & 7 & 58 & 10,0 & 68,7 & 16 \\
\hline 20 & 4,7 & 5 & 34 & 2,0 & 61,0 & 44 \\
\hline 21 & 4,6 & 7 & 38 & 3,0 & 64,6 & 41 \\
\hline 22 & 4,6 & 6 & 38 & 2,0 & 64,0 & 41 \\
\hline
\end{tabular}

${ }^{1} \mathrm{CTC}$ - capacidade de troca de cátions $\left(\sum \mathrm{K}^{+}, \mathrm{Ca}^{2+}, \mathrm{Mg}^{2+}, \mathrm{H}^{+}+\mathrm{Al}^{3+}\right)$.

$\mathrm{V} \%=$ saturação por bases. meio do solvente hexano (Instituto Adolfo Lutz, 1985). A produção de óleo por planta foi calculado baseandose na quantidade de lipídio total presente na amêndoa e na polpa multiplicado pela porcentagem dessas no fruto e pela massa desses por planta.

Os resultados após obtenção dos lipídios totais da amêndoa e da polpa foram submetidos à análise da variância (ANOVA) para detectar efeito de local e dentro de local de amostragem, sendo aplicando o teste de Tukey para comparação de médias, quando o teste $\mathrm{F}$ a $5 \%$ de probabilidade (Pimentel Gomes e Garcia, 2002).

$\mathrm{Na}$ quantificação do efeito de fertilidade do solo e dos atributos bromatológicos dos frutos, foi realizada a análise de componentes principais considerando como variáveis o $\mathrm{pH}, \mathrm{P}$ disponível, acidez potencial $\left(\mathrm{H}^{+}+\mathrm{Al}^{3+}\right)$, capacidade de troca de cátions (CTC), saturação por bases $(\mathrm{V} \%), \mathrm{Al}^{3+}$ trocável, quantidade de amêndoas no fruto (Am_fruto), teor de lipídios na amêndoa (Lip_Am), quantidade de polpa no fruto (Pol_fruto), teor de lipídios na polpa (Lip_pol) e produtividade de óleo por planta (*Prod). A matriz contendo todos os dados amostrais foi padronizada, para cada $j$ fixo, pela equação 1 . O poder discriminatório de cada variável foi avaliado pela equação 2 , enquanto a percentagem da variância total contida em cada componente $\mathrm{CP}_{\mathrm{h}}$ foi obtida segundo a equação 3 .

Equação 1: $Z_{k j}=\frac{X_{k j}-\bar{X}_{j}}{S_{j}}$

em que $\mathrm{k}$ é a amostra; $j$ é a característica; $\bar{X}_{\mathrm{j}}$ é a média; $\mathrm{S}_{\mathrm{j}}$ é o desvio padrão da coluna $j$.

Equação 2: $r_{x_{j}}\left(C P_{h}\right)=a_{j h} \frac{\sqrt{\lambda_{j}}}{S_{j}}$

em que $r_{x j}\left(\mathrm{CP}_{h}\right)$ é a correlação entre a variável $x_{j}$ e o componente principal $\mathrm{CP}_{h}$; $\mathrm{a}_{\mathrm{jh}}$ é o coeficiente da variável $j$ no $h$-ésimo componente principal; $\ddot{e}_{h}$ é o $h$-ésimo autovalor da matriz de covariância.

Equação 3: $C P_{h}=\frac{\lambda_{h}}{T(C)} 100$ em que:

$\mathrm{T}(\mathrm{C})$ é o traço da matriz de covariância $\left(\ddot{\mathrm{e}}_{1}+\ddot{\mathrm{e}}_{2}+\ldots+\ddot{\mathrm{e}}_{\mathrm{h}}\right)$. 
Anterior a aplicação do método multivariado de Análise de Componentes Principais (ACP) foram calculados os coeficientes de correlação de Pearson, pré-requisito este, exigido para aplicar a ACP, existência de correlação entre as variáveis sob pesquisa.

\section{Resultado e Discussão}

As porcentagens de lipídios totais na amêndoa e na polpa de frutos e a produtividade de óleo por planta estão apresentados na Tabela 3. Observou-se que os teores de lipídios totais das amostras de amêndoa foram influenciados pelos locais de amostragens com a área nove (BR 364) apresentando o maior valor $(22,8 \%)$, enquanto na oito na mesma rodovia a menor $(21,0 \%)$ menor porcentagem de lipídios, o que demonstra a presença de variabilidade entre plantas.

Apesar dessas variações, os teores médios ficaram mais de $100 \%$ abaixo dos encontrados por Ferreira et al. (2008) em frutos coletados no Estado do Amapá.
Fatores, como diversidade genética e condições diferentes de clima e solo devem ter interferido neste resultado (Schroth et al., 2004). Com relação ao teor de lipídios na polpa do tucumã, também houve variabilidade entre os locais amostrados (Tabela 3). Apenas os frutos coletados nos pontos 1, 2, 14, 17 e 18 apresentaram teores acima dos obtidos por Pesce (1941) no Estado do Pará e por Schroth et al. (2004) no Estado do Amazonas.

Os componentes principais 1 e 2 mostram a distribuição das amostras em conjunto com a distribuição das variáveis (Figura 1). A variabilidade retida nestes componentes explica $60,3 \%$ da variabilidade original, sendo que $\mathrm{CP}_{1}$ e $\mathrm{CP}_{2}$ retêm $37,7 \%$ e $22,6 \%$, respectivamente. No $\mathrm{CP}_{1}$ (eixo horizontal), as variáveis com maior poder discriminatório foram: $\mathrm{H}^{+}+\mathrm{Al}^{3+}(-0,93), \mathrm{Al}^{3+}(-0,87), \mathrm{V}$ $(0,85), \mathrm{pH}(0,81)$ e CTC $(-0,67)$, na qual cada valor contido entre parênteses representa a correlação entre cada variável e um componente principal. No caso do

Tabela 3. Porcentagem no fruto e lipídios totais na amêndoa e na polpa de frutos de tucumã

\begin{tabular}{|c|c|c|c|c|c|c|}
\hline Locais & Amostra & Am_fruto & Lip_Am $\%$ & Pol_fruto & Lip_pol & $\begin{array}{c}\text { Prod* } \\
\text { kg óleo/pl }\end{array}$ \\
\hline 1 & $\mathrm{~A}_{1-9,6}$ & 23,6 & $21,6 \mathrm{bc}$ & 59,0 & $33,5 \mathrm{a}$ & 9,6 \\
\hline 2 & $A_{2} \_6,1$ & 20,6 & $22,4 \mathrm{ab}$ & 44,8 & $33,6 \mathrm{a}$ & 6,1 \\
\hline 3 & $\mathrm{~A}_{3} \_2,7$ & 23,1 & $22,2 \mathrm{ab}$ & 43,6 & $30,9 \mathrm{~b}$ & 2,7 \\
\hline 4 & $A_{4-} 6,3$ & 23,6 & $22,3 \mathrm{ab}$ & 46,6 & $30,9 \mathrm{~b}$ & 6,3 \\
\hline 5 & $\mathrm{~A}_{5-} 3,0$ & 24,5 & $22,1 \mathrm{ab}$ & 39,2 & $31,5 \mathrm{~b}$ & 3,0 \\
\hline 6 & $\mathrm{~A}_{6-} 7,4$ & 22,8 & $22,6 \mathrm{ab}$ & 42,2 & $31,0 \mathrm{~b}$ & 7,4 \\
\hline 7 & $\mathrm{~A}_{7 \_} 2,8$ & 23,9 & $22,4 \mathrm{ab}$ & 46,5 & $31,3 \mathrm{~b}$ & 2,8 \\
\hline 8 & $\mathrm{~A}_{8-} 4,8$ & 23,7 & $21,0 \mathrm{c}$ & 46,5 & $31,4 \mathrm{~b}$ & 4,8 \\
\hline 9 & $\mathrm{~A}_{9 \_} \_3,0$ & 23,9 & $22,8 \mathrm{a}$ & 46,6 & $31,4 \mathrm{~b}$ & 3,0 \\
\hline 10 & $\mathrm{~A}_{10 \_} 2,4$ & 23,9 & $21,8 \mathrm{abc}$ & 46,5 & $31,3 \mathrm{~b}$ & 2,4 \\
\hline 11 & $\mathrm{~A}_{11-} 2,0$ & 23,8 & $21,9 \mathrm{abc}$ & 43,5 & $30,9 \mathrm{~b}$ & 2,0 \\
\hline 12 & $\mathrm{~A}_{12}-2,0$ & 26,8 & $22,2 \mathrm{ab}$ & 46,5 & $31,5 \mathrm{~b}$ & 2,0 \\
\hline 13 & $\mathrm{~A}_{13}-2,3$ & 24,0 & $22,1 \mathrm{ab}$ & 46,4 & $31,0 \mathrm{~b}$ & 2,3 \\
\hline 14 & $\mathrm{~A}_{14-2,1}$ & 25,6 & $22,1 \mathrm{ab}$ & 44,9 & $35,0 \mathrm{a}$ & 2,1 \\
\hline 15 & $\mathrm{~A}_{15-} 1,0$ & 23,8 & $22,0 \mathrm{abc}$ & 46,5 & $31,5 \mathrm{~b}$ & 1,0 \\
\hline 16 & $\mathrm{~A}_{16-} 0,8$ & 24,3 & $22,4 \mathrm{ab}$ & 46,4 & $31,5 b$ & 0,8 \\
\hline 17 & $\mathrm{~A}_{17-} 4,2$ & 27,1 & $21,6 \mathrm{bc}$ & 44,7 & $34,3 \mathrm{a}$ & 4,2 \\
\hline 18 & $\mathrm{~A}_{18-} 4,7$ & 25,7 & $22,1 \mathrm{ab}$ & 50,8 & $34,9 \mathrm{a}$ & 4,7 \\
\hline 19 & $A_{19-6,3}$ & 26,2 & $22,1 \mathrm{ab}$ & 47,4 & $31,3 \mathrm{~b}$ & 6,3 \\
\hline 20 & $\mathrm{~A}_{20-} 3,6$ & 24,8 & $22,2 \mathrm{ab}$ & 38,6 & $30,6 \mathrm{~b}$ & 3,6 \\
\hline 21 & $\mathrm{~A}_{21-} 4,9$ & 23,6 & $22,4 \mathrm{ab}$ & 47,4 & $31,1 \mathrm{~b}$ & 4,9 \\
\hline 22 & $\mathrm{~A}_{22-} 5,2$ & 22,1 & $22,1 \mathrm{ab}$ & 55,7 & $31,3 \mathrm{~b}$ & 5,2 \\
\hline $\mathrm{CV}(\%)$ & - & - & 1,14 & - & 1,11 & - \\
\hline
\end{tabular}

${ }^{1}$ Médias seguidas por letras distintas na mesma coluna diferem entre si pelo teste de Tukey a 5\% de probabilidade 


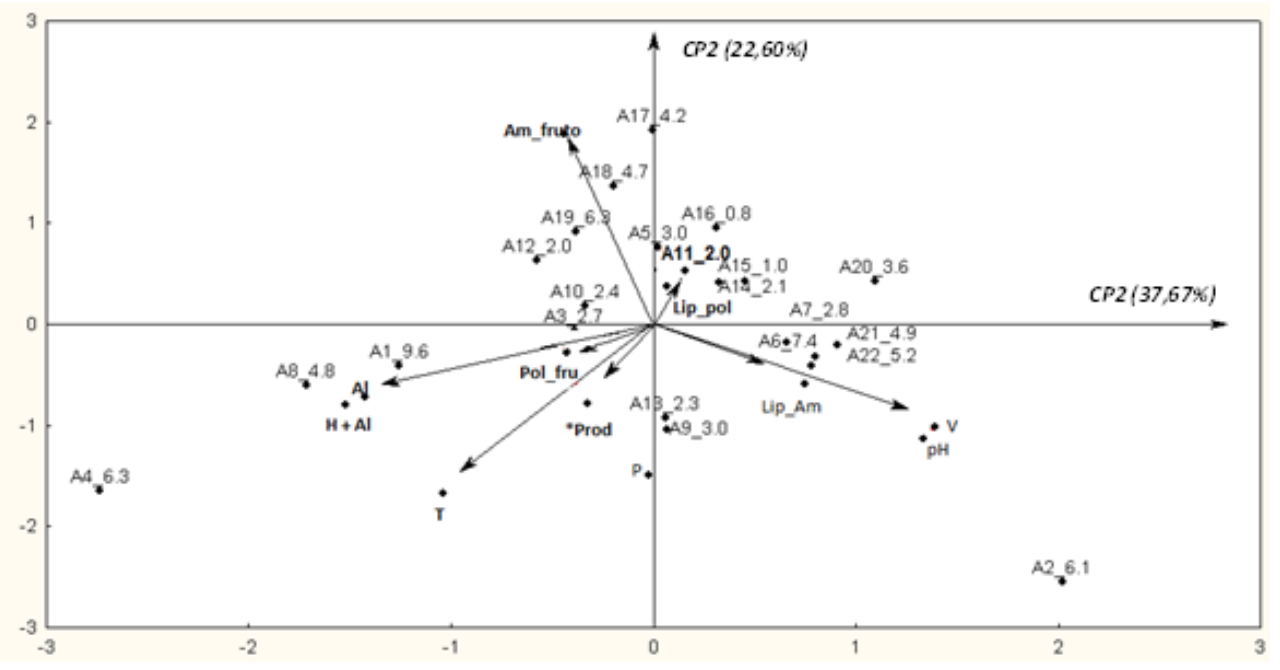

Figura 1. Gráfico bidimensional dos componentes principais 1 e 2 mostrando as distribuições das amostras e dos autovetores das variáveis analisadas.

$\mathrm{CP}_{2}$ (eixo vertical), as variáveis com maior poder discriminatório foram: Am_fruto $(0,79), \mathrm{T}(-0,70)$ e $\mathrm{P}$ $(-0,63)$, na qual, os valores contidos nos parênteses representam as correlações entre cada variável e um componente principal.

Considerando os dois eixos $\mathrm{CP}_{1}$ e $\mathrm{CP}_{2}$ ortogonais (Figura 1) tem-se a distribuição das amostras resumidas num plano bidimensional. As amostras que estão próximas à origem (0.0), não contribuem para o estudo, ou seja, não possuem características discriminatórias, indicando que os padrões dessas amostras estão dentro de uma média estatística e acompanham distribuição normal (Hicks, 1973). As amostras $\mathrm{A}_{1-} 9,6, \mathrm{~A}_{2}-6,1$, $\mathrm{A}_{4-} 6,3, \mathrm{~A}_{8-} 4,8, \mathrm{~A}_{18-} 4,7$ e $\mathrm{A}_{17-} 4,2$ se posicionaram dentro de uma região periférica tendo características próprias relevantes. Nas amostras localizadas abaixo do eixo, em geral, são aquelas com maior produtividade de óleo por planta distribuída tanto em solos com alta ou de baixa fertilidade (Figura 1). Na comparação com outras condições edafoclimáticas da Amazônia, os localizados no Estado do Acre são, na sua maior parte, os locais que apresentam as maiores fertilidades do solo (Bergo et al., 2006).

As amostras $A_{1-} 9,6, A_{4-} 6,3$ e $A_{8-} 4,8$ apresentaram boas produtividades em solo com elevada saturação por $\mathrm{Al}^{3+}$ trocável e $\mathrm{H}^{+}+\mathrm{Al}^{3+}$, enquanto a amostra $\mathrm{A}_{2} 6,1 \mathrm{em}$ solos com melhor fertilidade, caracterizado pelos altos valores de $\mathrm{pH}$ e saturação por bases (V\%). Os dados localizados na horizontal à esquerda e na vertical abaixo também apresentam boas produtividades (Figura 1). Tais resultados novamente demonstram a presença de grande variabilidade entre as plantas, como relatado por Schroth et al. (2004). Cabe destacar que se os vetores estão associados as variáveis respostas e se as variáveis num biplot apresentarem os vetores curtos e bem próximos da origem, estes não estão contribuindo com resposta positiva (Yan \& Tinker, 2006) para o incremento da

produtividade de amêndoas de tucumã.

De acordo com Barbosa et al. (2009), o tucumanzeiro pode ter boa produtividade em solos de baixa fertilidade natural. Essa característica é fator importante no manejo dessa cultura, visto o elevado custo dos fertilizantes e corretivos na região (Lehmann et al. 2001; Moreira e Gonçalves, 2006), dificulta a utilização destes produtos, principalmente, por pequenos produtores. Nos vetores da análise de componentes principais demonstram a associação entre os fatores P disponível, CTC, $\mathrm{H}^{+}+\mathrm{Al}^{3+}$, $\mathrm{Al}^{3+}$ trocável e a produtividade de óleo por planta. Assim, quanto mais abaixo for a posição da amostra, maior tendência que ocorra alta produtividade. Em contrapartida, as amostras que apresentam as maiores quantidades de amêndoa nos frutos (Am-fr) têm tendência a apresentar as menores produtividades.

Como o terceiro autovalor foi acima da unidade, temse ainda uma informação relevante a ser considerada, e assim pode ser construído o plano bidimensional formado pelos eixos $\mathrm{CP}_{1}$ e $\mathrm{CP}_{3}$ (Figura 2). A variabilidade retida nestes dois componentes explica $52,1 \%$ da variabilidade original, sendo que $\mathrm{CP}_{1}$ e $\mathrm{CP}_{3}$ retêm cada um, $37,7 \%$ e $14,4 \%$, respectivamente.

As variáveis com maior poder discriminatório foram: Lip_pol (-0,80), Pol_fruto $(-0,67)$, Assim, as amostras $A_{1-} 9,6, A_{2-} 6,1$ e $A_{8-} 4,8$ possuem maiores quantidades de polpa nos frutos e de lipídios na polpa, enquanto a $\mathrm{A}_{20}-3,6$ menores quantidades. De acordo com a Figura 2, verificou-se que as amostras $A_{1-} 9,6$, $\mathrm{A}_{8-} 4,8$ e $\mathrm{A}_{2-} 6,1$ possuem maior produtividade de óleo 


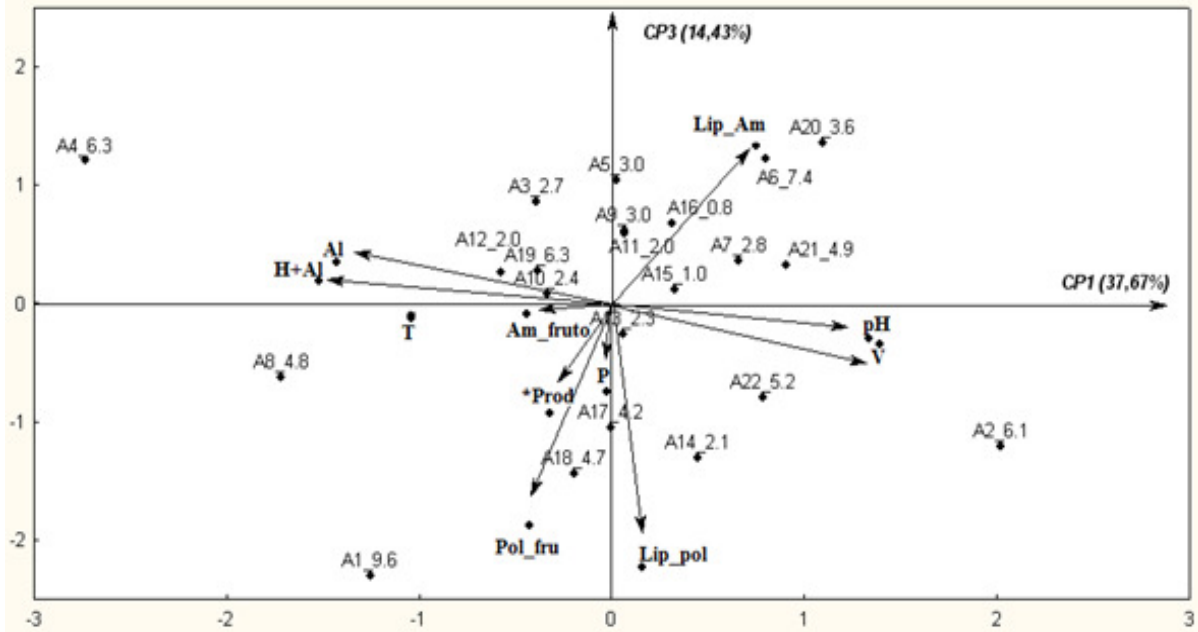

Figura 2. Gráfico bidimensional dos componentes principais 1 e 3 mostrando as distribuições das amostras e dos vetores das variáveis analisadas. fracassos das últimas décadas. Agrociências (Brasil) 9:67-71.

FERREIRA, E. S. et al. 2008. Caracterização físicoquímica do fruto e do óleo extraído de tucumã (Astrocaryum vulgare mart). Alimentos e Nutrição (Brasil) 19:427-433.

HICKS, C. R. 1973. Fundamental concepts in the design of experiments. New York, Holt, Rinehart \& Wilson. 349p.

INSTITUTO ADOLFO LUTZ. 1985. Normas de Análise do Instituto Adolfo Lutz. São Paulo, Instituto Adolfo Lutz. 533p. por planta e que os autovetores $\mathrm{CP}_{1}$ e $\mathrm{CP}_{3}$ apresentam associação entre a variável quantidade de polpa nos frutos e a produtividade de óleo por planta. Assim, amostras (plantas) localizadas abaixo do autovetor $\mathrm{CP}_{3}$ tendem a ter maior produtividade de lipídios.

\section{Conclusões}

A variabilidade genética e os atributos químicos do solo influenciaram na quantidade de lipídios totais contidos na amêndoa e na polpa.

O tucumanzeiro possui boa produtividade em óleo, mesmo em solos ácidos, de média a baixa fertilidade natural.

\section{Literatura Citada}

BACELAR-LIMA, C. G.; MENDONÇA, M. S.; BARBOSA, T. C. T. S. 2006. Morfologia floral de uma população de Tucumã, Astrocaryum aculeatum Meyer (Arecaceae) na Amazônia Central. Acta Amazonica (Brasil) 36(4):407-412.

BARBOSA, B. S. et al. 2009. Aproveitamento dos óleos de amêndoa de tucumã do Amazonas para produção de biodiesel. Acta Amazonica (Brasil) 39(2):371-376.

BERGO, C. L. et al. 2006. Avaliação de espécies leguminosas na formação de cafezais no segmento da agricultura familiar no Acre Acta Amazonica (Brasil) 36(1):19-24.

CAVAlCANTE, P. B. 1996. Frutas comestíveis da Amazônia. Belém, CNPq/Museu Paraense Emílio Goeldi. 279p.

CLEMENT, C. R.; ARKCOLL, D. B. 1991. The pejibaye (Bactris gasipaes H.B.K., Palmae) as an oil crop: potential and breeding strategy. Oléagineux (França) 46(7):293-299.

CLEMENT, C. R.; LLERAS PÉREZ, E.; van LEEUWEN, J. 2005. O potencial das palmeiras tropicais no Brasil: acertos e
LEHMANN, J. et al. 2001. Phosphorus management for crops in Central Amazonian upland soils. Plant and Soil 237:309-319.

LORENZI, H.; SOUZA, H. M.; MEDEIROS-COSTA, J. T. 1996. Palmeiras do Brasil: nativas e exóticas. Nova Odessa, Editora Plantarum. 306p.

MIRANDA, I. P. et al. 2001. Frutos de Palmeiras da Amazônia. Manaus, AM, Instituto Nacional de Pesquisa da Amazônia. 120p.

MENDONÇA, M. S. 1996. Aspectos morfológicos das sementes de algumas espécies de palmeiras (Arecaceae $=$ Palmae $) \mathrm{da}$ Amazônia. Tese Concurso de Professor Titular, Universidade do Amazonas, Manaus, AM. 68p.

MOREIRA, A.; GONÇALVES, J. R. P. 2006. Available phosphorus and potassium status of soils of Amazonas State. Better Crops with Plant Food 90:21-23.

PESCE, C. 1941. Oil palms and other oilseeds of the Amazon. Belém, PA, Revista de Veterinária(Brasil). 131p.

PEREIRA, F. E. A. 2007. Biodiesel produzido a partir do óleo de sementes de Mabea fistulifera. Dissertação Mestrado. Viçosa, MG, Universidade Federal deViçosa. 87p.

PIMENTEL GOMES, F.; GARCIA, C. H. 2002. Estatística aplicada a experimentos agronômicos e florestais. Piracicaba, SP, FEALQ. 309p.

RAIJ, B. et al. 1997. Recomendações de adubação e calagem para o Estado de São Paulo. Campinas, SP, Instituto Agronômico. 285p.

SHANLEY, P.; MEDINA, G. 2005. Frutíferas e plantas úteis na vida amazônica. Belém, PA,CIFOR. 300p.

SCHROTH, G. et al. 2004. Extractive use, management and in situ domestication of a weedy palm, Astrocaryum tucuma, in the central Amazon. Forest Ecology and Management 202:161-179.

YAN, W.; TINKER, N. A. 2006. Biplot analysis of multienvironment trial data: principles and applications. Canadian Journal of Plant Science 86: 623-645 